\title{
DESCRIÇÃO DE TÉCNICA DE REDUÇÃO CIRÚRGICA DAS LUXAÇÕES FACETÁRIAS DA COLUNA CERVICAL BAIXA POR VIA ANTERIOR
}

\author{
DESCRIPTION OF SURGICAL TECHNIQUE FOR REDUCTION OF FACET DISLOCATIONS \\ OF THE LOWER CERVICAL SPINE BY ANTERIOR APPROACH
}

\author{
DESCRIPCIÓN DE LA TÉCNICA QUIRÚRGICA PARA REDUCCIÓN DE LAS LUXACIONES \\ FACETARIAS DE LA COLUMNA CERVICAL BAJA POR ACCESO ANTERIOR
}

André Rafael Hübner ${ }^{1}$, Álvaro Diego Heredia Suárez², Jean Marcel Dambrós', Leandro de Freitas Spinelli ${ }^{3}$

\begin{abstract}
RESUMO
O trabalho descreve uma técnica cirúrgica de redução anterior das luxações facetárias da coluna cervical e discute as indicações para cirurgia por via anterior para as luxações da coluna cervical baixa. A técnica descrita neste artigo oferece excelentes resultados, conforme revisão bibliográfica e dos resultados do Serviço, tendo sido aplicada em até 95\% dos casos de fraturas-luxações. Não será abordada a apresentação de resultados neste trabalho, apenas a descrição e discussão da técnica aberta por via anterior. Observações de quarenta e um pacientes tratados nos últimos dez anos por esta técnica demonstram bons resultados quanto a pós-operatório menos doloroso, recuperação funcional extremamente rápida e complicações pouco frequentes.
\end{abstract}

Descritores: Traumatismos da coluna vertebral/cirurgia; Luxações; Vértebras cervicais;

\begin{abstract}
This paper describes a surgical technique for anterior reduction of the spinal facets dislocations and discusses its indications for surgery of lower cervical dislocations by anterior approach. The technique described in this article provides excellent results according to literature review and the results of the Service, having been applied to 95\% of cases of fracture-dislocations. The results of this work will not be presented, but the description and discussion of the open technique by anterior approach. Observations of 41 patients treated in last 10 years have shown amazing results as less postoperative pain, extremely rapid functional recovery, and lower complication rates.
\end{abstract}

Keywords: Spinal injuries/surgery; Dislocations; Cenvical vertebrae.

\section{RESUMEN}

El artículo describe una técnica quirúrgica para las luxaciones facetarias de la columna cervical y discute las indicaciones para la cirugía de luxación de la columna cervical baja por lo acceso anterior. La técnica descrita en este artículo proporciona excelentes resultados según la revisión de la literatura y los resultados del Servicio, después de haber sido aplicado a 95\% de los casos de fracturas-luxaciones. No serán abordados resultados, sino que únicamente la descripción y discusión de la técnica de reducción abierta por acceso vía anterior. Las observaciones en cuarenta y un pacientes operados en los últimos diez años por esta técnica muestran resultados sorprendentes con respecto a un pos operatorio menos doloroso, con recuperación funcional extremadamente rápida y complicaciones menos frecuentes.

Descriptores: Traumatismos vertebrales/cirugía; Luxaciones; Vértebras cervicales.

\section{INTRODUÇÃO}

Cerca de 14.000 lesões medulares ocorrem anualmente nos Estados Unidos e a maioria delas envolve a região cervical e inclui as fraturas-luxações. Neste mesmo período de tempo, milhares de outros pacientes sofrem trauma cervical sem lesão medular ${ }^{1}$. A Organização Mundial da Saúde - OMS considera os acidentes de trânsito como um dos maiores problemas de saúde pública do mundo. No Brasil, em 2001, morriam em torno de 18 pessoas vítimas desses acidentes por cada 100 mil habitantes. Os homens eram as maiores vítimas, com $29,9 \%$ de mortes contabilizadas contra 6,5\% entre as mulheres. Em termos regionais, os maiores índices foram encontrados nas regiões Centro-Oeste e Sul². Em 2008, enquanto os Estados Unidos obtiveram uma taxa de 12,5\% de mortes a cada 100.000 habitantes, o Brasil obteve uma taxa de $30,1 \%$, sendo que a frota de carros norte americana é o triplo da brasileira, ocorrendo, portanto, um aumento importante em relação a 2001. A maioria das vítimas fatais do trânsito no Brasil continua sendo homens jovens. A insuficiência de dados estatísticos fiéis à realidade é um obstáculo ao desenvolvimento de estratégias de intervenção adequadas e concretas ${ }^{3}$.

As ações de resgate que envolvem as vítimas devem estar fundamentadas no princípio de preservar o alinhamento do corpo, impedindo movimentos de torção, tanto no resgate como no transporte dos pacientes. O movimento poderá determinar um trauma irreversível na medula, provocado por fragmentos ósseos da vértebra, que podem seccionar, comprimir ou lacerá-la por completo4.

O tratamento das luxações da coluna cervical continua sendo um grande desafio para o cirurgião apesar dos avanços tecnológicos. Façanha Filho et al. ${ }^{5}$ apresentam um histórico da osteossíntese da fratura cervical: o primeiro relato foi feito por $\mathrm{Hadra}^{6}$ em 1891, utilizando amarrias; Rogers ${ }^{7}$ em 1942 propôs o uso de amarrias interespinhosas e assim o método tornou-se cada vez mais popular;

1. Preceptor do Serviço de Cirurgia da Coluna do Instituto de Ortopedia eTraumatologia de Passo Fundo - Passo Fundo, RS, Brasil.

2. Estagiário (R5) em Cirurgia de Coluna do Instituto de Ortopedia eTraumatologia de Passo Fundo - Passo Fundo, RS, Brasil.

3. Medico Assistente do Serviço de Ortopedia eTraumatologia da Irmandade da Santa Casa de Misericórdia de Porto Alegre - Porto Alegre, RS, Brasil.

Trabalho realizado no Instituto de Ortopedia eTraumatologia de Passo Fundo, Hospital São Vicente de Paulo e Clínica Kozma - Passo Fundo, RS, Brasil. Correspondência: Rua Uruguai, 2050, Cep 99010-112, Passo Fundo/ RS, Brasil. arhubner@terra.com.br e scc@iotrs.com.br 
Burke e Berrymon ${ }^{8}$ em 1971 descreveram o uso da redução incruenta com paciente sob anestesia geral, seguida de imobilização externa; Roy-Camille e Saillant ${ }^{9}$ em 1979 relatam o uso de placas e parafusos como meio de fixação das lesões traumáticas da coluna cervical e defenderam o uso desta técnica para obtenção de maior estabilidade. Cooper et al. ${ }^{10}$ em 1988 revisaram 20 pacientes tratados com uso de placas e parafusos posteriores e concluíram que este meio seria superior às amarrias. Oliveira ${ }^{11}$ em 1979 apresentou a descrição da redução anterior para as luxações facetárias em 12 pacientes, sendo 10 unilateral e 2 bilaterais, relatando ser uma técnica fácil e segura, sem complicações significativas.

O manejo básico das luxações da coluna cervical baixa não mudou muito nos últimos 10 anos. A tração axial com objetivo de reduzir e alinhar as articulações de forma temporária continua sendo o procedimento de emergência mais utilizado, porém ainda discutido. O melhor método de redução e estabilização ainda é controverso e o cirurgião pode decidir a forma de tratamento. A cirurgia pode incluir estabilização por via anterior, posterior ou combinadas ${ }^{12}$. A via anterior apresenta baixa morbidade, fácil posicionamento na mesa cirúrgica, além de oferecer acesso ao disco intervertebral quando deslocado pelo trauma, o qual pode causar compressão medular secundária ${ }^{13}$.

O tratamento não cirúrgico com tração halocraneana seguida de imobilização externa acabou demonstrando desvantagens quando comparado com o tratamento cirúrgico. Portanto, o tratamento conservador vem caindo em desuso devido à demora na recuperação dos pacientes, perda do alinhamento e deformidade residual em cifose $^{14,15}$

Através do tratamento cirúrgico com a reconstrução da anatomia cervical e com estabilização interna, tem-se um período de recuperação mais rápido do paciente com o retorno às atividades diárias. Definido que o tratamento cirúrgico pode ser a melhor opção, surge a segunda questão para o cirurgião: qual é a técnica cirúrgica mais adequada e qual a via de abordagem a ser utilizada ${ }^{16}$.

O presente trabalho descreve em detalhes a técnica cirúrgica de redução via anterior para as luxações facetárias da coluna cervical utilizada no Instituto de Ortopedia e Traumatologia de Passo Fundo/ RS e Hospital São Vicente de Paulo, realizada com êxito há 10 anos.

\section{MÉTODOS}

A técnica a ser descrita e discutida é utilizada pelo Serviço de Cirurgia da Coluna do Instituto de Ortopedia e Traumatologia de Passo Fundo/RS (IOT/RS) e Hospital São Vicente de Paulo (HSVP) desde 2000 e foram operados 41 pacientes até o momento. Como protocolo geral de atendimento, os pacientes eram avaliados na chegada à emergência pelo Advanced Trauma Life Support (ATLS) e posteriormente através de exame neurológico específico, envolvendo o Índice de Lucas e Ducker ${ }^{17}$ para avaliação da força muscular e a Escala de Frankel et al. ${ }^{18}$ para a avaliação da deficiência sensitivo-motora. Os pacientes realizaram radiografias da coluna cervical em incidências anteroposterior, trans-oral e perfil (visualizando-se desde a base do crânio ao platô superior de T1 e, em caso de não visualização, solicitava-se a posição do nadador, ou ainda tomografia computadorizada (TC), especialmente em pacientes desorientados ou inconscientes). Se as radiografias eram de boa qualidade e não apresentavam alterações, o paciente era liberado com colar cervical Philadelphia em caso de dor e marcado retorno ao ambulatório em 15 dias para pesquisa de instabilidade ligamentar com RX dinâmicos. Confirmado o diagnóstico de luxação, e não havendo TC anteriormente da emergência, realizava-se então o exame para a classificação do tipo de lesão. A ressonância nuclear magnética (RNM) não faz parte do protocolo de rotina da emergência deste Serviço, mas é realizada em casos de suspeita de lesão ligamentar, hematoma medular, SIWORA (Spinal Injury Without Radiological Abnormalities) se houver radiografias normais ou em caso de comprometimento neurológico ${ }^{19}$ Utilizamos como rotina a classificação de Argenson et al. ${ }^{20}$ e Allen et al. ${ }^{21}$.

\section{Técnicas Cirúrgicas}

Nos pacientes que apresentam luxações uni ou bi facetarias, ou fratura luxação, segundo a classificação de Argenson et al. ${ }^{20} \mathrm{e}$ Allen et al. ${ }^{21}$, mantém-se a imobilização segundo o protocolo ATLS. Após avaliadas as condições clinicas do paciente, realizamos duas tentativas de redução fechada no centro cirúrgico sob controle do intensificador de imagem, mantendo o paciente com colar cervical tipo Philadelphia temporariamente até o procedimento cirúrgico de artrodese objetivando o tratamento definitivo. Em caso de não se conseguir a redução fechada inicialmente, realiza-se a redução e o procedimento cirúrgico no mesmo tempo, com discectomia e artrodese do segmento lesado por via anterior ${ }^{22}$.

\section{Redução Fechada}

A redução fechada é realizada através de tração axial seguida de flexão cervical com pinça de Gardner-Wells a $2 \mathrm{~cm}$ acima do vértice das orelhas e a $1 \mathrm{~cm}$ atrás deste ponto ${ }^{22,23}$, com aumento progressivo de peso de $4,5 \mathrm{~kg}$ para C1, elevando-se o peso em $5 \mathrm{~kg} /$ nível, tendo como limite $32 \mathrm{~kg}$ de acordo com critérios de White e Panjabi ${ }^{23,24}$. Devem-se realizar controles radiológicos a cada 30 minutos após cada colocação de peso, com paciente lúcido, sendo que o procedimento não deve demorar mais de 2 horas. A pinça é colocada sob anestesia local, e a tração é realizada com manobras de flexão da coluna cervical, tração axial, rotação para o mesmo lado da luxação ampliando o espaço interfacetário afetado quando a luxação é unilateral, e estende-se o pescoço até zero grau ou leve hiperextensão (Figura 1). Obtida a redução, é realizada a discectomia do nível lesado e artrodese anterior com placa e enxerto tricortical de ilíaco. Caso não seja obtida a redução através das manobras especificas em até duas tentativas com o paciente acordado, realizamos o procedimento com anestesia geral e no mesmo tempo realizamos a discectomia e artrodese via anterior com placa e enxerto tricortical de ilíaco. Caso o paciente apresente piora neurológica ou alterações dos sinais vitais durante o procedimento, devem ser suspensas imediatamente as manobras e ser realizada a discectomia primariamente para posterior obtenção da redução.

\section{Redução Aberta}

A redução aberta é realizada com paciente em decúbito dorsal com um coxim na região interescapular para se obter uma leve extensão do pescoço e realizar tração dos membros superiores para distal ao longo do corpo, fixando-os com fita adesiva para uma boa visualização da coluna cervical com o intensificador de imagens (fluoroscopia transoperatória). Opta-se pela anestesia geral com relaxantes musculares, importantes para o auxílio na redução da luxação. A técnica utilizada neste trabalho é semeIhante à proposta por Oliveira ${ }^{11}$, mas com algumas modificações. Utiliza-se o acesso anterior tipo Smith-Robinson, localiza-se de lesão através de auxílio fluoroscópico, realiza-se a ressecção do ligamento longitudinal anterior, faz-se a discectomia total do nível de lesão e, em seguida, há a ressecção do ligamento longitudinal posterior, permitindo as manobras de redução e fixação anterior. Realiza-se tração axial e flexão com pinça de Gardner-Wells e se realizam manobras rotacionais especificas de acordo com o lado da faceta luxada (Figura 1). Todo o processo é monitorizado pelo uso de fluoroscopia após a discectomia. Posiciona-se o gancho de Cloward no ângulo superior da vértebra inferior no muro posterior - canal vertebral - à vértebra atingida, com objetivo de fazer uma alavanca aumentando a luxação e tracionando-se o gancho para anterior (Figura 2). Utiliza-se como apoio o dedo polegar do cirurgião na face anterior da vértebra do nível imediatamente superior e faz-se compressão de anterior para posterior para ajudar na redução ao mesmo tempo mantendo-se a tração constante com a pinça de Gardner-Wells durante as manobras. Quando se obtém a redução faz-se a extensão do pescoço (leva-se da flexão para a hiperextensão). Realizam-se testes de flexão e extensão com a fluoroscopia para controlar a estabilidade.

Em caso de não se conseguir a redução, tenta-se novamente 


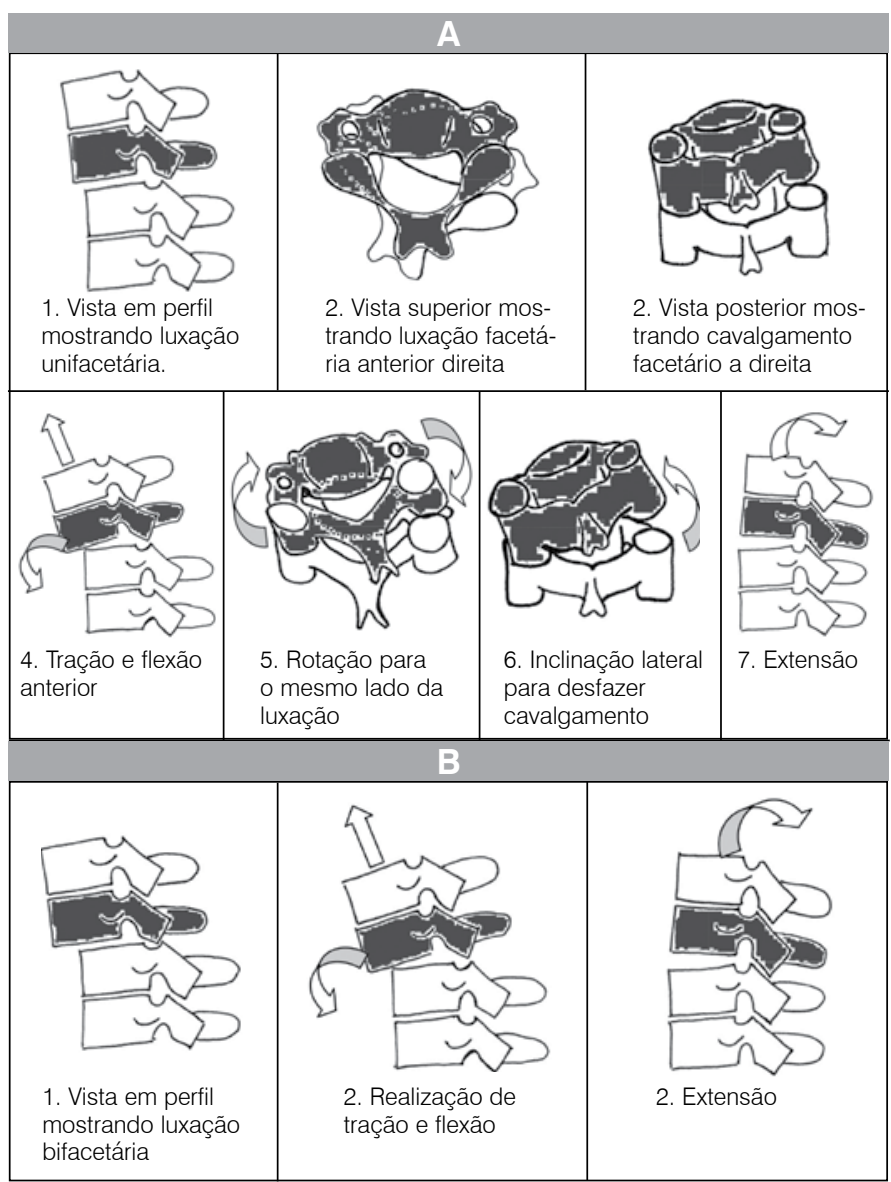

Figura 1. Manobras de redução das luxações cervicais: (A) unifacetária e (B) bifacetária.

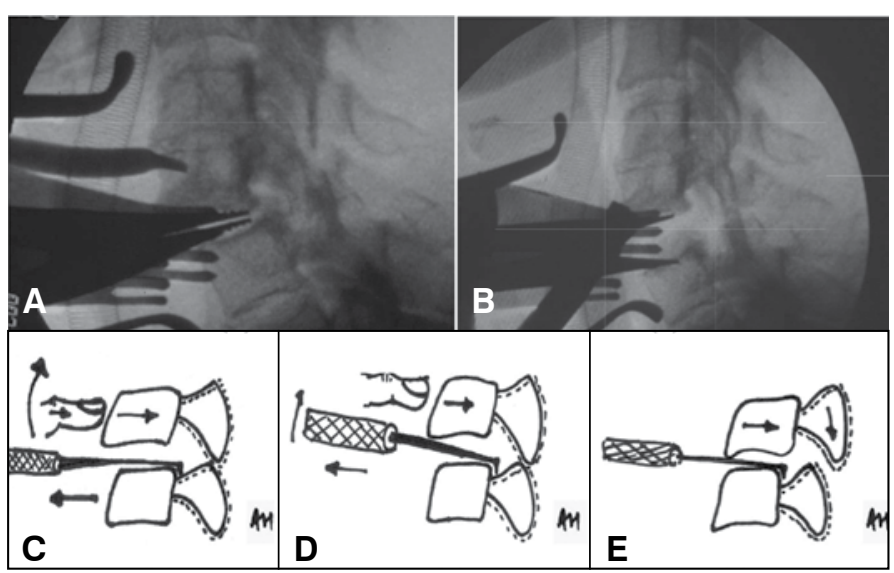

Figura 2. (A) Tração axial após discectomia e (B) tração axial e flexão 45 graus com objetivo de desimpactar as facetas articulares; (C) tração axial e extensão com ajuda de gancho de Cloward para levar a vértebra inferior para anterior e (D) compressão anterior da vértebra superior para completar a redução, baixando-se o ganho para posterior remoção (E).

o procedimento. Quando não se consegue a redução adequada, considera-se existência de bloqueio por fragmentos da faceta, massa ou pedículo, e então pode ser realizado o procedimento cirúrgico associado à via posterior ou, se houver menos de $25 \%$ de desalinhamento da linha anterior do corpo vertebral, podemos fazer a fixação "in situ". Cabe aqui salientar que a fixação "in situ" de luxações com amplitudes de $25 \%$ é controversa. A obtenção da redução de forma total nem sempre é possível. Nestes casos, a fixação "in situ" pode se tornar uma opção, pois apresenta bons resultados segundo a literatura.
Após a redução ser obtida, o procedimento deve ser capaz de restabelecer a estabilidade, a qual se perde pela lesão e ruptura de tecidos (cápsula articular, disco, ligamentos), que podem levar a um possível novo deslocamento em caso de não haver fixação. Para evitar que haja nova luxação durante o procedimento, mantém-se extensão do pescoço até que a fixação seja efetuada. Realiza-se então a artrodese com auto-enxerto tri-cortical de ilíaco fixado com placa e parafusos nas vértebras superior e inferior, buscando a fixação em duas corticais por cada parafuso (18 a 22mm) quando possível, e imediatamente após a fixação faz-se flexão e extensão para testar a estabilidade obtida no nível e identificar outras instabilidades adjacentes e avaliar a qualidade da fixação (Figura 3). Utiliza-se como rotina um dreno fechado à vácuo de $3.2 \mathrm{~mm}$.

Utilizamos o colar tipo Philadelphia por um período de 8 a 12 semanas no pós-operatório. Controles ambulatoriais clínicos e radiográficos são realizados aos 15 dias, 45 dias, três meses, seis meses e após anualmente para se pesquisar movimento no nível de fusão, falha de material, consolidação do enxerto e degeneração de níveis adjacentes.

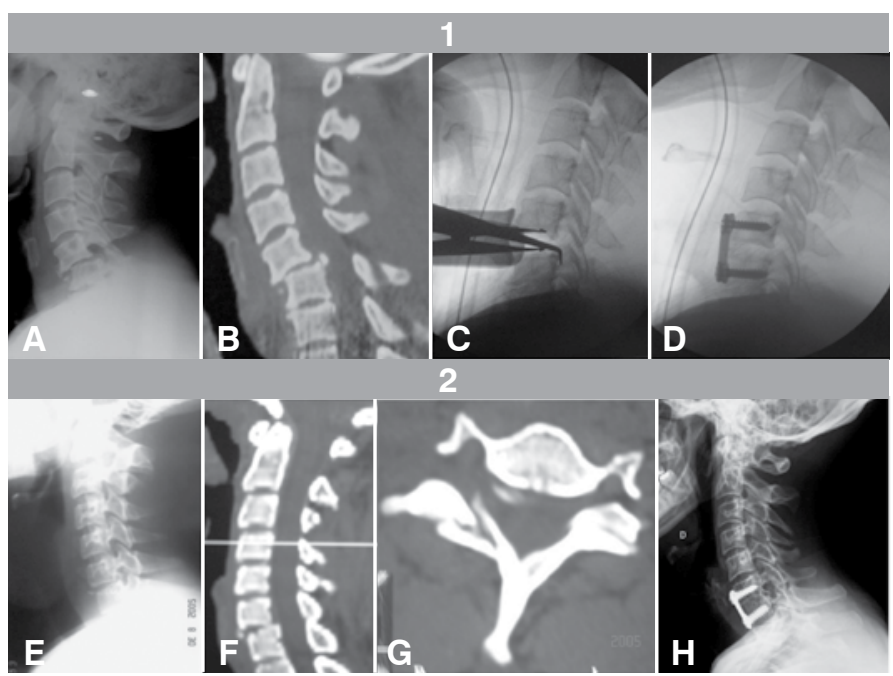

Figura 3. (1) Paciente masculino com 47 anos de idade, vítima de acidente de carro, luxação bifacetária C5-C6, Frankel E, tratado cirurgicamente pela técnica descrita. (A) radiografia em perfil mostrando luxação bifacetária com fratura-avulsão do ligamento longitudinal anterior; (B) TC com reconstrução sagital; (C) fluoroscopia intra-operatória mostrando mecanismo de redução com gancho de Cloward; (D) fluoroscopia intra-operatória mostrando artrodese por via anterior com placa, parafusos e auto-enxerto de ilíaco; (2) Paciente feminina com 40 anos de idade com fratura-luxação unifacetária C6-C7, Frankel D, tratada cirurgicamente pela técnica descrita. (E) radiografia em perfil mostrando luxação unifacetária com fratura-avulsão do ligamento longitudinal anterior; (F) TC com reconstrução sagital; (G) TC em corte axial mostrando luxação unifacetária associada com fratura da faceta esquerda; $(H)$ radiografia pós-operatória tardia mostrando artrodese por via anterior com placa, parafusos e auto-enxerto de ilíaco.

\section{DISCUSSÃO}

O manejo das luxações da coluna cervical baixa ainda é controverso. O tempo para a realização da cirurgia depende do estado clínico geral do paciente, observando-se a evolução do estado neurológico e o tipo de acesso nos casos de tratamento cirúrgico depende da estratégia, preferência e treinamento do cirurgião. Bons resultados já foram publicados após estudos biomecânicos para fixação com placa e parafusos por via posterior e anterior. $\mathrm{O}$ acesso anterior para esse tipo de lesões tem se tornado cada vez mais popular, devido a sua fácil abordagem, mínima lesão de tecido muscular, visualização da herniação e ressecção de herniações migradas, aumentando a segurança no momento das manobras com a visualização direta e também demonstra resultados satisfatórios com baixo índice de complicações ${ }^{25,26}$.

Oliveira ${ }^{11}$ analisou 12 pacientes operados através da técnica de redução anterior e relatou que é uma técnica fácil, segura e sem complicações significativas. Não foi observada deterioração neuro- 
lógica em nenhum paciente. Um destes pacientes, que apresentava inicialmente Síndrome de Brown-Sequard, recuperou-se completamente em 4 meses. Nesta pesquisa também não foi observada deterioração nervosa dos pacientes atendidos e operados pelo Serviço de Coluna do IOT/PF.

Vital et al. ${ }^{25}$ publicaram uma série de 163 pacientes operados pela técnica da via anterior. Não houve complicações com deterioração neurológicas em nenhum dos casos após a manobra de redução fechada ou aberta com discectomia. Os autores referem ter tido dificuldade de redução em apenas cinco casos. Reindl et al. ${ }^{27}$ apresentaram uma série de 41 casos com resultados similares e referem que taxas de sucesso da técnica variam de 30 a $100 \%{ }^{25,28,29}$. Ordonez et al. ${ }^{30}$ encontraram $90 \%$ de sucesso na redução, descompressão e fusão. Johnson et al. ${ }^{31}$ apresentaram $13 \%$ de taxas de perda de redução associada à presença de quebra do implante ou da faceta. Nesta pesquisa não encontramos complicações referentes ao procedimento de tração pré-operatório ou ao procedimento aberto via anterior, e houve 100\% de redução.

Argenson et al. ${ }^{32}$ avaliaram as luxações traumáticas rotatórias da coluna cervical baixa em 47 pacientes com seguimento de 6 meses a 7 anos. Em 30\% dos casos os autores observaram radiculopatias. O protocolo terapêutico utilizados pelos autores foi o mesmo para praticamente todos os pacientes (90\%), com redução seguida de artrodese anterior com placa e parafusos utilizando enxerto de ilíaco. Nos demais pacientes, foi necessária abordagem posterior por dificuldades de redução. Em nossa série de 41 casos, realizamos dupla abordagem para apenas um caso $(2,4 \%)$, por luxação inveterada e com anquilose das facetas em subluxação.

Lee et al. ${ }^{13}$ discutem que a via anterior apresenta baixa morbidade e oferece bom acesso ao disco intervertebral, estando este deslocado pelo trauma na maioria dos casos e referem que pode causar compressão medular ${ }^{13}$. Grauer et al. ${ }^{33}$ referem que a ressonância magnética pode trazer alguma informação adicional ao cirurgião, no diagnostico de hérnia de disco traumática cervical mas acarreta atrasos na redução da luxação em função da demora para autorizar e executar o exame, apresenta variações de interpretação e claramente não altera a tomada de decisão sobre cirurgia ou tratamento conservador ${ }^{33}$. Entretanto, estudos clínicos falharam em demonstrar correlação entre tempo de redução e o quadro neurológico final ${ }^{34}$. Portanto, existe pouca evidência para se tomar uma decisão entre iniciar logo o tratamento fechado da luxação facetária, obter ressonância magnética pré-operatória ou proceder diretamente o tratamento cirúrgico aberto ${ }^{31,35}$

O protocolo do Serviço de Cirurgia da Coluna IOT/RS e HSVP, depois de confirmado o diagnóstico de luxação da coluna cervical baixa através de radiografias e TC, é a instalação de tração axial com pinça de Gardner-Wells na admissão do paciente ao hospital, realizando manobras específicas na tentativa de reduzir a luxação facetária o mais breve possível, levando em conta o estado clínico do paciente e, em casos de não ser possível a redução, opta-se pela cirurgia via anterior pelo acesso de Smith-Robinson, discectomia, redução da lesão e artrodese com auto-enxerto tri-cortical e placa em "H". O procedimento tem demonstrado excelentes resultados.

\section{CONCLUSÃO}

A técnica cirúrgica por via anterior nas fraturas-luxações facetárias demonstrou ser uma técnica segura com resultados clínicos satisfatórios e com baixo índice de complicações no grupo de 41 pacientes operados até o presente momento. Este acesso vem se tornando muito frequente na prática ortopédica com objetivo de descomprimir a medula, diminuir o risco de compressão secundária por herniação discal após redução, reduzir a luxação e restabelecer a estabilidade. Apesar de haver outra via de acesso para as luxações, a técnica da via anterior vem sendo utilizada com sucesso, além de possibilitar todo o procedimento em somente um tempo e em única via.

\section{REFERÊNCIAS}

1. Hadley MN, Walters BC, Grabb PA, Oyesiku NM, Przybylski GJ, Resnick DK, ET AL. Guidelines for the management of acute cervical spine and spinal cord injuries. Clin Neurosurg. 2002;49:407-98.

2. Organización Mundial de la Salud (OMS). Informe mundial sobre prevención de los traumatismos causados por el transito. [Resumen]. Ginebra; 2004.

3. Confederação Nacional de Municípios. Mapeamento das Mortes por Acidentes de Trânsito no Brasil. Distrito Federal: Brasília; 2010. (Estudos CNM Técnicos).

4. Cloward R. Treatment of acute fracture and fracture dislocations of the cervical spine by vertebral body fusion. J Neurosurgery. 1961;18:201-9.

5. Façanha Filho, FAM, Kusabara R, Avanzi O. Amarria interespinhosa como síntese nas fraturas da coluna cervical de C3 -T1. Rev Bras de Ortop. 1994;29(6):401-10

6. Hadra BE. Wiring of the vertebrae as a means of immobilization in fracture and Potts' disease. Med Times Register. 1891;22:423-9.

7. Rogers WA. Treatment of fracture-dislocation of the cervical spine. J Bone Joint Surg. 1942;24:245-58

8. Burke DC, Berryman D. The place of closed manipulation in the management of flexionrotation dislocations of the cervical spine. J Bone Joint Surg Br. 1971;53(2):165-82.

9. Roy-Camille R, Saillant G. Chirurgie du rachis cervical inferieur. Techniques d' osteosynthese dur rachis cervical inferiour. In: Premieres Journées de la Pitie, Paris, Masson; 1979. p. 36-50.

10. Cooper PR, Cohen A, Rosiello A, Koslow M. Posterior stabilization of cervical spine fractures and subluxations using plates and screws. Neurosurgery. 1988;23(3):300-6.

11. Oliveira JC. Anterior reduction of interlocking facets in the lower cervical spine. Spine. 1979;4(3):195-202

12. Montesano PX, Jnach E. Anatomic and biomechanical study of posterior cervical spine plate arthrodesis. Orthop Trans. 1989:13:205-306.

13. Lee TT, Alameda GJ, Camilo E, Green BA. Surgical treatment of post-traumatic myelopathy associated with syringomyelia. Spine (Phila Pa 1976). 2001;26(24 Suppl):S119-27.

14. Nassr A, Lee JY, Dvorak MF, Harrop JS, Dailey AT, Shaffrey Cl, et al. Variations in surgical treatment of cervical facet dislocations. Spine (Phila Pa 1976). 2008;33(7):E188-93.

15. Defino HLA, Fuentes AER, Russo NJ. Osteossíntese das lesões traumáticas da coluna cervical baixa (C3-C7). Rev Bras Ortop. 1994:29(3):127-35

16. Ratliff JK, Lebude B, Albert T, Anene-Maidoh T, Anderson G, Dagostino P, et al. Complications in spinal surgery: comparative survey of spine surgeons and patients who underwent spinal surgery. J Neurosurg Spine. 2009;10(6):578-84.

17. Lucas JT, Ducker TB. Motor classification of spinal cord injuries with mobility, morbidity and recovery indices. Am Surg. 1979;45(3):151-8.

18. Frankel HL, Hancock DO, Hyslop G, Melzak J, Michaelis LS, Ungar GH, et al. The value of postural reduction in the initial management of closed injuries of the spine with paraplegia and tetraplegia. I. Paraplegia. 1969;7(3):179-92.

19. Grauer JN, Vaccaro AR, Lee JY, Nassr A, Dvorak MF, Harrop JS, et al. The timing and

influence of MRI on the management of patients with cervical facet dislocations remains highly variable: a survey of members of the Spine Trauma Study Group. J Spinal Disord Tech. 2009;22(2):96-9.

20. Argenson $C$, De Peretti $F$ Eude $P$ Classification des lésions traumatiques du rachis cervical inférieur. Cahiers d'enseignement de la SOFCOT. 2000;76:42-61.

21. Allen BL Jr, Ferguson RL, Lehmann TR, O'Brien RP. A mechanistic classification of closed, indirect fractures and dislocations of the lower cervical spine. Spine (Phila Pa 1976). 1982;7(1):1-27

22. Sack JA, Etame AB, Shah GV, La Marca F, Park P. Management and outcomes of patients undergoing surgery for traumatic cervical fracture-subluxation associated with an asymptomatic vertebral artery injury. J Spinal Disord Tech. 2009;22(2):86-90.

23. Lerman JA, Dickman CA, Haynes RJ. Penetration of cranial inner table with Gardner-Wells tongs. J Spinal Disord. 2001;14(3):211-3.

24. White AA, Southwick WO, Panjabi MM. Clinical instability in the lower cervical spine. A review of past andcurrent concepts. Spine. 1976;1:15-27.

25. Vital JM, Gille O, Sénégas J, Pointillart V. Reduction technique for uni- and biarticular dislocations of the lower cervical spine. Spine (Phila Pa 1976). 1998;23(8):949-54.

26. Robertson PA, Ryan MD. Neurological deterioration after reduction of cervical subluxation. Mechanical compression by disc tissue. J Bone Joint Surg Br. 1992;74(2):224-7.

27. Reindl R, Ouellet J, Harvey EJ, Berry G, Arlet V. Anterior reduction for cervical spine dislocation. Spine (Phila Pa 1976). 2006;31(6):648-52

28. Grant GA, Mirza SK, Chapman JR, Winn HR, Newell DW, Jones DT, et al. Risk of early closed reduction in cervical spine subluxation injuries. J Neurosurg. 1999:90(Suppl 1):13-8.

29. Star AM, Jones AA, Cotler JM, Balderston RA, Sinha R. Immediate closed reduction of cervical spine dislocations using traction. Spine (Phila Pa 1976). 1990;15(10):1068-72.

30. Ordonez BJ, Benzel EC, Naderi S, Weller SJ. Cervical facet dislocation: techniques for ventral reduction and stabilization. J Neurosurg. 2000;92(Suppl 1):18-23

31. Johnson MG, Fisher CG, Boyd M, Pitzen T, Oxland TR, Dvorak MF. The radiographic failure of single segment anterior cervical plate fixation in traumatic cervical flexion distraction injuries. Spine (Phila Pa 1976). 2004;29(24):2815-20.

32. Argenson C, Lovet J, Sanouiller JL, de Peretti F. Traumatic rotator displacement of the lower cervical spine. Spine (Phila Pa 1976). 1988;13(7):767-73.

33. Grauer JN, Vaccaro AR, Lee JY, Nassr A, Dvorak MF, Harrop JS, et al. The timing and influence of MRI on the management of patients with cervical facet dislocations remains highly variable: a survey of members of the Spine Trauma Study Group. J Spinal Disord Tech. 2009:22(2):96-9.

34. Greg Anderson D, Voets C, Ropiak R, Betcher J, Silber JS, Daffner S, et al. Analysis of patient variables affecting neurologic outcome after traumatic cervical facet dislocation. Spine J. 2004;4(5):506-12.

35. Sabiston CP, Wing PC, Schweigel JF, Van Peteghem PK, YuW. Closed reduction of dislocations of the lower cervical spine. J Trauma. 1988;28(6):832-5. 